\title{
THERAPEUTIC EXERCISE IN CHRONIC LOW BACK PAIN
}

\author{
Florina Ojoga, UMF Carol Davila, \\ Smaranda Marinescu
}

\begin{abstract}
Rehabilitation of patients with low back pain is very important because the incidence of this symptom is very high among all ages, but especially after forty years. The rehabilitation team must perform a detailed clinical exam and an assessment of the functional limitations and functional goals to achieve at the end of the treatment for every patient. The goals of the therapy are normalization of impairments in flexibility, strength and endurance, and of course, reducing of pain.
\end{abstract}

KEY-WORDS: low back pain, therapeutic exercise, functional improvement.

\section{INTRODUCTION}

Low back pain is a symptom, not a disease, and has many causes, being described as pain between the costal margin and the gluteal folds. It is extremely common because studies had shown a lifetime prevalence as high as $84 \%$.

Onset usually begins in the teens to early forties and most patients have short attacks of pain that are mild to moderate and do not limit activities, but tend to recur over many years. A small percentage of low back pain becomes chronic and causes significant disability (1).

The percentage of patients disabled by low back pain, as well as the costs for it, have steadily increased during the last 30 years. The two most commonly factors are the increasing social acceptance of back pain as a reason to become disabled and the social system that pays benefits to patients with this pathology (2).

\section{PATHOLOGIC CHANGES IN LOW BACK PAIN}

Degeneration of the structures of the spine is associated with aging, being accelerated in patients with previous trauma or injury to the lumbar spine. The most commonly involved levels are L4-L5 and L5-S1, because there are the greatest torsion and compressive loads.
The zygapophyseal joints may develop osteoarthritis. The intervertebral disk experiences progressive dehydration in the normal aging process of the spinal structures. Fissures in the annulus fibrosus may develop, causing an inflammatory response and further degeneration may result in the progression of the disease or complete annular tears, which provoke discogenic low back pain, also named internal disk disruption syndrome (39\% of the patients with low back pain).

The loss of segmental integrity may lead to further degeneration of the disk, with narrowing of the intervertebral disk space. Because of increased loads on the posterior elements, facet arthropaty may develop, a source of low back pain. The loss of disk height may cause subluxation of the facet joints, resulting in degenerative spondylolisthesis, most commonly at the L4-L5 level.

Other factors associated with lumbar degeneration include environmental, occupational and psychosocial influences. Environmental factors include cigarette smoking and occupational activities that involve repetitive bending, sitting or vibrational stresses. Psychosocial factors are well known to contribute to significant disability in low back pain. 


\section{FUNCTIONAL LIMITATIONS IN LOW BACK PAIN}

Functional limitations in degenerative diseases of the lumbar spine depend on the anatomic structures involved. All aspects of daily living, including self-care, work, sports activities and recreation may be affected (2).

Symptoms are typically exacerbated during bending, twisting and forward flexion in patients with primary discogenic pain.

Patients with facet arthropathy or instability report increased pain with extension-based activities, including standing and walking. Pain is often relieved with sitting and forward-flexed positions.

Patients with myofascial or soft tissue syndromes report pain that is worsened with static and prolonged physical activity. Symptomatic improvement is associated with rest and modalities as heat, cold and pressure.

Patients with contributing psychological factors, such as depression and somatization disorders, report pain out of proportion to the underlying pathologic process, poor sleep and significant disability in their daily activities (3).

\section{REHABILITATION TREATMENT}

The most important treatment of any low back pain is education and reassurance of the patient. Most of the acute low back pain symptoms are self-limited and resolve in 4-6 weeks. Therapy is directed toward management of the symptoms rather than the disease.

Initial therapy for lumbar degenerative disease should consist of antiinflammatory medications, muscle relaxants, occasionally opioid medications for severe symptom exacerbation and a functionally oriented physical therapy program. Most patients feel well with these measures and do not require invasive procedures. Other useful initial treatments may include trigger point injections as well as heat and cold modalities. Low-dose tricyclic antidepressants can help with improvement of sleep (1).

Rehabilitation of lumbar degenerative disc disease includes a thorough assessment of functional limitations and functional goals for each patient. A full assessment of occupational and recreational activities demands and goals should also be made.

Occasionally, lumbar orthotics can be prescribed, but these are not beneficial in the treatment of the degenerative disc disease unless there is a significant spondylolisthesis or some other specific indications.

The treatment focus on normalization on impairments in flexibility, strength and endurance and of course, on healthy lifestyle modifications. Physical modalities such as ultrasound and electrical therapy can be used for the acute cases, however, the focus of therapy should be on an active program rather than on the passive treatments (3).

\section{Exercise}

Multiple studies have found that exercise results in positive outcomes in the treatment of chronic low back pain, including pain relief (although modest, with a mean difference of 10 points on a 100 point scale), improvement in function and slightly reduced sick-leave.

The most effective exercise for low back pain includes an individualized treatment learned and performed under the supervision of a therapist that includes stretching and strengthening. The purpose of exercises for the treatment of low back pain is to strengthen and increase endurance of muscles that support the spine and improve flexibility in areas that have stiffness. These exercises are combined with motor retraining to establish normal patterns of motor activity (4). 
The exact dose and number of exercises and the ideal length of treatment is not known. Because endurance is a significant problem with many patients with chronic low back pain, activity levels should be increased in function of realistic goals rather than on symptoms. Beyond the physiologic benefits of exercise, increasing activity has positive effects on behaviors about pain. Adverse effects of exercise for low back pain are rarely reported, so is a very safe form of treatment.

\section{Specific exercise treatment for low back pain}

Exercise prescriptions for mechanical low back pain begin with the goal of improving alignment and posture, because the exercises are more effective if they are done from a position of proper alignment that promotes optimal joint function and movement patterns (6).

Many postural faults begin as habitual, and then become structural as tight muscles and tendons do not allow immediate correction, and weak muscles cannot maintain the proper position. This happens in long-standing lordosis, in which hip flexors and lumbar paraspinals become tight from prolonged positioning in lordosis, and abdominal muscles become long and weak from disuse and lengthened position. These types of faults can be addressed with the proper exercises to stretch tight areas and strengthen weak areas (7).

Lumbar stabilization and core strengthening exercises that strengthen the muscles that support the spine are the most common exercises used to treat low back pain. Because the deep stabilizers (multifidi and transversus abdominus) do not function well, some programs begin with the training of these muscles. Then the exercises progress to more complex dynamic and functional tasks, sometimes called motor control exercises because the precision of movement is the goal, rather than simply gaining global strength or flexibility (8).

\section{Aquatic exercises for mechanical low back pain}

Patients who have not tolerated land-based exercises are often able to participate in pool exercises, which have several benefits: buoyancy and reduction of gravitational stress. The greater the amount of the body is submerged, the greater the effect. There is a reduction of $90 \%$ in gravitational stresses when the patient is in vertical position and is immersed in water to the neck(5).

Water can decrease pain and muscle overactivity is also decreased in warm water. The same principles for progressing therapy apply to aquatic exercise as to land-based exercise. Patients can learn neutral position, stabilizing and other strengthening exercises, and by walking, jogging or swimming can add an aerobic component (9).

Multiple studies have found a beneficial effect on pain and function for patients with low back pain who exercise in the water.

\section{PROGNOSIS OF LOW BACK PAIN}

Prognosis is difficult to ascertain for many reasons. One is that low back pain is a symptom caused by a large spectrum of pathology with many prognostic outcomes and another is that the pain experience is individual and treatment expectations may vary. There are many complex cultural, psychologic and economic factors that influence pain and rehabilitation outcome.

After rehabilitation treatment, most patients are improving their function within 1 month, and the most continue to have pain decrease, although more slowly, until about 3 months. From 3 months to 1 year, little change in pain is seen. The risk of recurrence within 3 months is between $19 \%$ and $34 \%$, and increases after 1 year between $66 \%$ and $84 \%$ (6). 


\section{CONCLUSION}

Exercise has generally been found to be one of the most effective treatments for decreasing pain and increasing function in chronic low back pain. The many other health benefits of exercise, along with the low risk of causing harm, make it a firstline treatment for mechanical low back pain.

\section{BIBLIOGRAPHY}

1.Kirkaldy-Willis WH. Managing low back pain. New York, Churchill Livingstone, 1999.

2.Rainville J, Ahern DK, Phalen L. The association of pain with physical activities in chronic low back pain. Spine 1992; 17:1060-1064.

3.Saal JA. Dynamic muscular stabilization in the nonoperative treatment of lumbar pain syndromes. Orthop Rev 1990; 19: 691-700.

4.Bliss LS, Teeple P. Core stability: the centerpiece of any training program. Current Sports Med Rep 2005; 4:179-183.

5.Konlian C. Aquatic therapy: making a wave in the treatment of low back pain. Orthop Nursing 18: 11-20,1999.

6.Liddle SD, Baxter GD. Exercise and chronic low back pain: what works? Pain 107:176-190,2004.

7.Macedo LG, Maher CG. Motor control exercise for persistent, nonspecific low back pain: a systematic review. Phys Ther 89: 9-25, 2009.

8.Richardson C, Jull G, Hodges P. General considerations in motor control and joint stabilization: the basis of assessment and exercise techniques. Therapeutic exercise for spinal segmental stabilization in low back pain. Edinburgh 1999, Churchill Livingstone.

9.Waller B, Lambeck J. Therapeutic aquatic exercise in the treatment of low back pain: a systematic review. Clin Rehabil 23 (1):3-14, 2009. 